\title{
Stable, high-order computation of traveling water waves in three dimensions
}

\author{
David P. Nicholls ${ }^{\mathrm{a}, *}$, Fernando Reitich ${ }^{\mathrm{b}}$ \\ a Department of Mathematics, University of Notre Dame, Notre Dame, IN 46556, USA \\ ${ }^{\mathrm{b}}$ School of Mathematics, University of Minnesota, Minneapolis, MN 55455, USA
}

Received 3 June 2005; received in revised form 11 November 2005; accepted 14 November 2005

Available online 10 January 2006

\begin{abstract}
The Euler equations of free-surface ocean dynamics constitute a model of central importance in fluid mechanics due to the wide range of physical phenomena they are intended to represent, from shoaling and breaking of waves in nearshore regions to energy and momentum transport in the open ocean. From a mathematical perspective, these equations present rather unique challenges for analysis and simulation as they couple the subtleties of nonlinear wave equations (balancing nonlinearity with dispersion in the absence of dissipation) to the difficulties of free-boundary problems. In this paper a new, stable high-order boundary perturbation algorithm for the numerical simulation of traveling water waves is described. Its performance is compared to that of classical surface deformation algorithms and it is shown that the new scheme displays significantly enhanced conditioning properties and a lower computational cost, which enable very accurate predictions of physical observables such as velocity, energy, height/steepness, and shape.
\end{abstract}

(c) 2005 Elsevier SAS. All rights reserved.

Keywords: Ideal fluids; Capillary-gravity waves; Traveling waves; Numerical simulation; Boundary perturbation; High-order methods; Spectral methods

\section{Introduction}

The Euler equations of free-surface ocean dynamics constitute a model of central importance in fluid mechanics due to the wide range of physical phenomena they are intended to represent, from shoaling and breaking of waves in nearshore regions to energy and momentum transport in the open ocean. From a mathematical perspective, these equations present rather unique challenges for analysis and simulation as they couple the subtleties of nonlinear wave equations (balancing nonlinearity with dispersion in the absence of dissipation) to the difficulties of free-boundary problems. In this paper, we describe a new, stable high-order boundary perturbation algorithm for the numerical simulation of traveling water waves that is based upon our recent theoretical study [1] of analyticity properties of solutions of the Euler equations. We compare its performance to classical surface deformation algorithms (which can

\footnotetext{
* Corresponding author.

E-mail address: nicholls@math.uic.edu (D.P. Nicholls).
} 
be traced to Stokes [2]) and we show that the enhanced speed, stability, and conditioning of our new schemes lead to very accurate predictions of physical observables such as velocity, energy, height/steepness, and shape.

There is a large literature concerning the numerical simulation of free-surface fluid flows. In the case of a $d$-dimensional $(d=2,3)$ ideal fluid (with $(d-1)$-dimensional surface), attention has centered on boundary integral/element methods (BIM/BEM) and "high-order spectral" (HOS) methods. Both approaches posit unknown surface quantities and, due to this reduction in dimension, they are generally preferred to volumetric methods. Tsai and Yue [3] provide an excellent and comprehensive overview of the state-of-the-art in this field up to the mid-1990's, primarily focused on the initial value problem. Since the appearance of this article, subsequent work has largely focused on simulations of three-dimensional flows. Notable among these are the BIM/BEM of Beale [4]; Grilli, Guyenne, and Dias [5]; Xue, Xu, Liu, and Yue [6]; and Liu, Xue, and Yue [7].

Regarding traveling free-surface ideal fluid flows, Dias and Kharif [8] provide a thorough overview of much of the current theory and computational approaches. Notable among the papers on numerical simulation of the full Euler equations are: Schwartz [9] who studied two-dimensional traveling patterns via complex variable theory, the boundary integral method of Schwartz and Vanden-Broeck [10], and the HOS three-dimensional simulations of Rienecker and Fenton [11]; Meiron, Saffman, and Yuen [12]; Roberts and Schwartz [13]; Saffman and Yuen [14]; and Bryant [15]. These latter authors all derive a nonlinear system of equations from the ideal fluid equations for unknown Fourier coefficients, and then find solutions by Newton iteration and/or numerical continuation. More recent calculations of Nicholls [16,17], and Craig and Nicholls [18] were achieved with a numerical continuation method applied to a Fourier spectral discretization of the Hamiltonian formulation of the water wave equations as posed by Zakharov [19].

The work that is most closely related to the method we advocate here is that of Roberts [20]; Roberts and Peregrine [21]; and Marchant and Roberts [22]. These are also HOS methods, but rather than solving a single set of nonlinear equations, they are based on the assumption that the velocity of the traveling wave, the wave shape, and the velocity potential all depend analytically upon a small parameter, say $\varepsilon$, and on the derivation of linear (inhomogeneous) equations for these quantities at every perturbation order. Once obtained, these quantities are summed at the desired value of $\varepsilon$. These methods can be viewed as iterative implementations of the HOS methods of [11-15] preconditioned by the linear traveling water waves problem; see Nicholls and Reitich [23]. While these methods can be quite useful within their domain of applicability, they suffer from several severe limitations. First, their computational complexity (execution time) scales poorly with discretization parameters. For truly two-dimensional surfaces [1], if $N$ orders are approximated with $N_{1} \times N_{2}$ spatial discretization points, the execution time is $\mathcal{O}\left(N^{3} N_{1}^{2} N_{2}^{2}\right)$. Additionally, as with other classical boundary perturbation methods (see Nicholls and Reitich [23-27]), these algorithms suffer from numerical instability at high order due to subtle but significant cancellations (see Section 3.1).

To improve upon these classical perturbative HOS we present here a new, high-order spectral algorithm for the stable and accurate approximation of traveling water waves. Our new scheme is also perturbative in nature. In contrast with the classical methodologies, however, our new procedures effect the perturbation expansions in appropriately transformed spatial coordinates. The choice of transformation (which effectively "flattens" the free boundary) is based upon our earlier theoretical work that demonstrated its beneficial effects within boundary perturbation algorithms. Indeed, as the results in [1] show, a suitable change of independent variables can have a rather dramatic effect on the conditioning of these methods. With regards to computational cost, and while the change of variables introduces source terms into the Euler equations demanding a volumetric discretization, a transparent boundary condition can be implemented which, as we shall show, permits the discretization in the vertical dimension to occur in a very small neighborhood of the free surface, so that it is effectively a surface discretization. Indeed, as we shall show, the resulting schemes display significant improvements in overall computational cost over classical perturbative HOS. More precisely, the computational cost is merely

$$
\mathcal{O}\left(N N_{1} N_{2} \log \left(N_{y}\right) N_{y}+N^{2} N_{1} N_{2} N_{y}\right)
$$

in execution time.

The structure of the paper is as follows: In Section 2 we set up our notation as we review the basic Euler model. In Section 3.1 we recall the method of Roberts et al. [20-22] which we shall refer to as "Field Expansions" (FE), and discuss its limited stability properties. Section 3.2 is devoted to the introduction of our new approach, the method of "Transformed Field Expansions". Three-dimensional numerical results are then presented in Section 4 which demonstrate the accuracy of the scheme in the computation of one-dimensional bifurcation branches of travelingwave solutions. In Section 5 we present results based on the extension of these algorithms to allow for the direct 
recovery of full two-dimensional bifurcation surfaces, whose existence was demonstrated in [1]. Finally, in Section 6 we present our conclusions.

\section{Governing equations}

The classical model for the free-surface evolution of an ideal (irrotational, inviscid, incompressible) fluid under the influence of gravity and capillarity is the Euler equations (see Lamb [28]). If the fluid occupies the domain

$$
S_{h, \eta}=\left\{(x, y) \in \mathbb{R}^{d-1} \times \mathbb{R} \mid-h<y<\eta(x, t)\right\},
$$

where $0<h \leqslant \infty$, then the irrotational nature of the flow implies that the fluid velocity is the gradient of a potential, $v=\nabla \varphi$, inside $S_{h, \eta}$. At the impermeable bottom the normal velocity is set to zero (in the case $h=\infty$ the $y$-component of velocity tends to zero as $y \rightarrow-\infty$ ), while in the horizontal directions we choose, for simplicity, the classical assumption of periodic boundary conditions with respect to the lattice $\Gamma \in \mathbb{R}^{d-1}$ and period cell $P(\Gamma)$. Finally, these are supplemented with initial conditions, and kinematic and Bernoulli relations at the free surface,

$$
\begin{array}{ll}
\partial_{t} \eta+\nabla_{x} \eta \cdot \nabla_{x} \varphi-\partial_{y} \varphi=0 & \text { at } y=\eta, \\
\partial_{t} \varphi+\frac{1}{2}|\nabla \varphi|^{2}+\left[g-\sigma \Delta_{x}\right] \eta-\sigma H(\eta)=0 & \text { at } y=\eta,
\end{array}
$$

where

$$
H(\eta)=\operatorname{div}_{x}\left[\frac{\nabla_{x} \eta}{\sqrt{1+\left|\nabla_{x} \eta\right|^{2}}}-\nabla_{x} \eta\right],
$$

$g$ is the constant of gravity, and $\sigma$ is the coefficient of surface tension. Traveling waves translating uniformly with speed $c \in \mathbb{R}^{d-1}$, therefore satisfy

$$
\begin{array}{ll}
\Delta \varphi=0, & -h<y<\eta(x), \\
\partial_{y} \varphi(x,-h)=0, & \\
{\left[c \cdot \nabla_{x}\right] \eta+\nabla_{x} \eta \cdot \nabla_{x} \varphi-\partial_{y} \varphi=0} & \text { at } y=\eta, \\
{\left[c \cdot \nabla_{x}\right] \varphi+\frac{1}{2}|\nabla \varphi|^{2}+\left[g-\sigma \Delta_{x}\right] \eta-\sigma H(\eta)=0} & \text { at } y=\eta .
\end{array}
$$

\subsection{A transparent boundary condition}

An equivalent and numerically advantageous restatement of (2) can be made in terms of a transparent boundary condition. To derive this condition we note that for $|\eta|_{L^{\infty}}<a<h$, (2) can be restated with the aid of an "augmented velocity potential", $\tilde{\varphi}$,

$$
\begin{array}{ll}
\Delta \varphi=0, & -a<y<\eta(x), \\
{\left[c \cdot \nabla_{x}\right] \eta+\nabla_{x} \eta \cdot \nabla_{x} \varphi-\partial_{y} \varphi=0} & \text { at } y=\eta, \\
{\left[c \cdot \nabla_{x}\right] \varphi+\frac{1}{2}|\nabla \varphi|^{2}+\left[g-\sigma \Delta_{x}\right] \eta-\sigma H(\eta)=0} & \text { at } y=\eta, \\
\Delta \tilde{\varphi}=0, & -h<y<-a, \\
\partial_{y} \tilde{\varphi}(x,-h)=0, & \\
\varphi(x,-a)=\tilde{\varphi}(x,-a), & \\
\partial_{y} \varphi(x,-a)=\partial_{y} \tilde{\varphi}(x,-a) . &
\end{array}
$$

Eqs. (3d) and (3e) have solution

$$
\tilde{\varphi}(x, y)=\sum_{k \in \Gamma^{\prime}} a_{k} \cosh (|k|(y+h)) \mathrm{e}^{\mathrm{i} k \cdot x},
$$


where $\Gamma^{\prime}$ is the conjugate lattice to $\Gamma$. With (3f) in mind we note that for a general Dirichlet condition at $y=-a$, say

$$
\tilde{\varphi}(x,-a)=\xi(x) \equiv \sum_{k \in \Gamma^{\prime}} \hat{\xi}_{k} \mathrm{e}^{\mathrm{i} k \cdot x},
$$

the solution becomes

$$
\tilde{\varphi}(x, y)=\sum_{k \in \Gamma^{\prime}} \hat{\xi}_{k} \frac{\cosh (|k|(y+h))}{\cosh (|k|(h-a))} \mathrm{e}^{\mathrm{i} k \cdot x} .
$$

Furthermore, Neumann data, cf. (3g), can be computed as

$$
v(x)=\partial_{y} \tilde{\varphi}(x,-a)=\sum_{k \in \Gamma^{\prime}} \hat{\xi}_{k}|k| \tanh (|k|(h-a)) \mathrm{e}^{\mathrm{i} k \cdot x}
$$

Letting $D=-\mathrm{i} \nabla_{x}$, the right-hand side of (5) can be used to define an "order-one pseudodifferential operator":

$$
T \equiv|D| \tanh ((h-a)|D|),
$$

whose action on the Fourier series (4) is given by

$$
T \xi=\sum_{k \in \Gamma^{\prime}} \hat{\xi}_{k}|k| \tanh ((h-a)|k|) \mathrm{e}^{\mathrm{i} k \cdot x} .
$$

This definition then allows for an equivalent statement of (3) in the form

$$
\begin{array}{ll}
\Delta \varphi=0, & -a<y<\eta(x), \\
\partial_{y} \varphi(x,-a)-T \varphi(x,-a)=0, & \\
{\left[c \cdot \nabla_{x}\right] \eta+\nabla_{x} \eta \cdot \nabla_{x} \varphi-\partial_{y} \varphi=0} & \text { at } y=\eta, \\
{\left[c \cdot \nabla_{x}\right] \varphi+\frac{1}{2}|\nabla \varphi|^{2}+\left[g-\sigma \Delta_{x}\right] \eta-\sigma H(\eta)=0} & \text { at } y=\eta .
\end{array}
$$

This alternative statement not only allows one to focus on a considerably truncated problem domain, but also provides a uniform statement of the water wave problem for any depth including infinite depth (note that if $h=\infty$ then $T=|D|)$.

\subsection{Bifurcation theory}

We seek non-trivial traveling wave solutions of the Euler equations via bifurcation theory. A trivial branch of solutions exists at the "quiescent state", $\varphi=\eta=0$ and any velocity $c$, and we search for solutions nearby. This theory requires an analysis of the linearization of (6) about these quiescent solutions:

$$
\begin{array}{ll}
\Delta \bar{\varphi}=0, & -a<y<0, \\
\partial_{y} \bar{\varphi}(x,-a)-T \bar{\varphi}(x,-a)=0, & \\
{\left[\bar{c} \cdot \nabla_{x}\right] \bar{\eta}-\partial_{y} \bar{\varphi}=0} & \text { at } y=0, \\
{\left[\bar{c} \cdot \nabla_{x}\right] \bar{\varphi}+g \bar{\eta}-\sigma \Delta_{x} \bar{\eta}=0} & \text { at } y=0 .
\end{array}
$$

In the case of finite depth, (7a), (7b), and the periodic boundary conditions imply that

$$
\bar{\varphi}(x, y)=\sum_{k \in \Gamma^{\prime}} \bar{\varphi}_{k} \frac{\cosh (|k|(y+h))}{\cosh (h|k|)} \mathrm{e}^{\mathrm{i} k \cdot x}, \quad \bar{\eta}(x)=\sum_{k \in \Gamma^{\prime}} \bar{\eta}_{k} \mathrm{e}^{\mathrm{i} k \cdot x} .
$$

Eqs. (7c) and (7d) mandate

$$
A(\bar{c}, k)\left(\begin{array}{c}
\bar{\varphi}_{k} \\
\bar{\eta}_{k}
\end{array}\right) \equiv\left(\begin{array}{cc}
-|k| \tanh (h|k|) & \mathrm{i} \bar{c} \cdot k \\
\mathrm{i} \bar{c} \cdot k & g+\sigma|k|^{2}
\end{array}\right)\left(\begin{array}{c}
\bar{\varphi}_{k} \\
\bar{\eta}_{k}
\end{array}\right)=\left(\begin{array}{l}
0 \\
0
\end{array}\right),
$$

for all $k \in \Gamma^{\prime}, k \neq 0$. Clearly, a non-trivial solution exists only if $A(\bar{c}, k)$ is singular, i.e. if the determinant

$$
\Lambda_{\sigma}(\bar{c}, k)=(\bar{c} \cdot k)^{2}-\left(g+\sigma|k|^{2}\right)|k| \tanh (h|k|)
$$


is zero. If a pair $(\bar{c}, \bar{k})$ satisfies $\Lambda_{\sigma}(\bar{c}, \bar{k})=0$ then a solution of (7) is

$$
\begin{aligned}
& \bar{\eta}(x)=\alpha(\bar{c} \cdot \bar{k}) \mathrm{e}^{\mathrm{i} \bar{k} \cdot x}+c . c ., \\
& \bar{\varphi}(x, y)=\alpha \mathrm{i}\left(g+\sigma|\bar{k}|^{2}\right) \frac{\cosh (|\bar{k}|(y+h))}{\cosh (h|\bar{k}|)} \mathrm{e}^{\mathrm{i} \bar{k} \cdot x}+c . c .,
\end{aligned}
$$

where $\alpha \in \mathbf{C}$. The character of these linear solutions can be revealed by writing $\alpha=(\rho / 2) \mathrm{e}^{\mathrm{i} \theta}$ and simplifying:

$$
\begin{aligned}
& \bar{\eta}(x)=\rho(\bar{c} \cdot \bar{k}) \cos (\bar{k} \cdot x+\theta), \\
& \bar{\varphi}(x, y)=\rho\left(g+\sigma|\bar{k}|^{2}\right) \frac{\cosh (|\bar{k}|(y+h))}{\cosh (h|\bar{k}|)} \sin (\bar{k} \cdot x+\theta) .
\end{aligned}
$$

These solutions are clearly two-dimensional waveforms in a rotated set of coordinates. To obtain truly $d$-dimensional nonlinear waves we begin by selecting $(d-1)$-many linearly independent wavenumbers $\kappa_{1}, \ldots, \kappa_{d-1} \in \Gamma^{\prime}$ and then solve $\Lambda_{\sigma}\left(\bar{c}, \kappa_{j}\right)=0$ for $\bar{c} \in \mathbb{R}^{d-1}$, i.e. $K \bar{c}=R$, where $K \in \mathbb{R}^{(d-1) \times(d-1)}$ has rows $\kappa_{j}$, and

$$
R_{j}= \pm \sqrt{\left(g+\sigma\left|\kappa_{j}\right|^{2}\right)\left|\kappa_{j}\right| \tanh \left(h\left|\kappa_{j}\right|\right)}
$$

we can, without loss of generality, choose the signs of the $R_{j}$ so that $\bar{c}_{j} \geqslant 0$. In this case, the most general linear solution will be a sum of terms, as in (10), with $\bar{k}=\kappa_{j}$ :

$$
\begin{aligned}
& \bar{\eta}(x)=\sum_{j=1}^{d-1} \rho_{j}\left(\bar{c} \cdot \kappa_{j}\right) \cos \left(\kappa_{j} \cdot x+\theta_{j}\right), \\
& \bar{\varphi}(x, y)=\sum_{j=1}^{d-1} \rho_{j}\left(g+\sigma\left|\kappa_{j}\right|^{2}\right) \frac{\cosh \left(\left|\kappa_{j}\right|(y+h)\right)}{\cosh \left(h\left|\kappa_{j}\right|\right)} \sin \left(\kappa_{j} \cdot x+\theta_{j}\right) .
\end{aligned}
$$

Note that, without loss of generality, we may choose $\theta_{j}=0$ (by effecting a suitable translation). Moreover, to construct nonlinear solutions which linearize to (11), it suffices to consider, upon reparameterization, the case $\rho_{1}=1$.

We note here that an important consideration in this analysis is the possibility that there may be other wavenumbers $\kappa_{d}, \ldots, \kappa_{p}$ such that $\Lambda_{\sigma}\left(\bar{c}, \kappa_{j}\right)=0$. In fact if $\sigma=0$ then $p$ can be infinite thereby exhibiting the phenomenon of "small divisors" (see Craig and Nicholls [29]). This is an instance of resonance in the traveling wave problem and currently lies outside the scope of our theory (see [1]).

\section{Boundary perturbation methods}

In this section we review the basic ideas and equations behind the new perturbative approach we introduced in [1] for the theoretical analysis of bifurcating traveling wave solutions. These theoretical developments suggest that a numerical scheme based on this novel formulation should lead to significantly more stable simulations than those based on classical perturbative approaches [20-22]. As we show in Section 4, this is indeed the case and, moreover, these higher quality solutions can be obtained at a comparatively lower computational cost. For the sake of completeness we begin the discussion here with a brief review of the classical approach in Section 3.1, where we also re-examine the origins and manifestation of its ill-conditioning; the details of our new, stable formulation then follow in Section 3.2.

\subsection{Field expansions and cancellations}

A classical approach of Stokes [2] to approximating solutions of (6) consists of the boundary perturbation philosophy we have termed "Field Expansions" - FE - (to distinguish it from the alternative "Operator Expansions" approach, see e.g. Watson and West [30]; West, Brueckner, Janda, Milder, and Milton [31]; Milder [32]; and Craig and Sulem [33]) carried out to order one or two. This method was extended to higher orders by subsequent researchers with the most recent developments by Roberts et al. [20-22]. This method posits that the field $\varphi$, surface $\eta$, and speed $c$ can be expanded in convergent Taylor series:

$$
\varphi(x, y, \varepsilon)=\sum_{n=1}^{\infty} \varphi_{n}(x, y) \varepsilon^{n}, \quad \eta(x, \varepsilon)=\sum_{n=1}^{\infty} \eta_{n}(x) \varepsilon^{n}, \quad c(\varepsilon)=\sum_{n=0}^{\infty} c_{n} \varepsilon^{n} .
$$


Then (2) is used to provide differential equations for $\left\{\varphi_{n}, \eta_{n}, c_{n}\right\}$ at every order on the simple geometry $S_{h, 0}$. The interested reader is referred to [1] for a detailed description and explicit formulas.

As we noted in [1], the derivation of the FE recursions is purely formal in nature. Indeed to justify this procedure it must be shown that the expansions (12) converge in some sense. An examination of the explicit recurrences defining the coefficients in (12) (see [1]), however, reveals that this can only be accomplished by estimating (in an appropriate function space) the spatial derivatives of the velocity potential, $\varphi_{n}$, at increasingly high orders and applying the triangle inequality. However, as we have shown in related boundary perturbation approaches (see Nicholls and Reitich [23-27]), these bounds cannot establish a finite rate of growth in any norm of these solutions since they destroy substantial cancellations which underly the FE recursions.

Following [1], we substantiate this claim within the present context with a careful two-dimensional numerical experiment. Naturally, the two-dimensional setting is chosen since moderately sized values of $N_{1}$ are required to display the cancellations; when $N_{j}$ is even somewhat large the $\mathcal{O}\left(N^{3} N_{1}^{2} N_{2}^{2}\right)$ cost of the three-dimensional FE algorithm is prohibitive. If we choose $\sigma=0, h=\infty, g=1,2 \pi$-periodicity, and $\kappa_{1}=3$, then a bifurcating family of traveling waves emanates from $c_{0}=1 / \sqrt{3}$. We present results of an FE implementation up to order $N=30$ with $N_{1}=256$ so that all convolution products (performed using Fast Fourier acceleration in vectors of length $N_{1}$, representing wavenumbers $\left.\left[-N_{1} / 2, N_{1} / 2-1\right]\right)$ are free of aliasing.

Motivated by physical considerations, we compute the $L^{2}$-norm of the wave form $\eta(x)$ which measures the potential energy of the wave, cf. (29). More specifically, if we denote by

$$
\eta_{N}^{N_{1}}(x ; \varepsilon)=\sum_{n=0}^{N} \sum_{k=-N_{1} / 2}^{N_{1} / 2-1} \hat{\eta}_{n}(k) \mathrm{e}^{\mathrm{i} k x} \varepsilon^{n},
$$

which represents the FE approximation to $\eta(x)$, then in Fig. 1 we present the difference, measured in $L^{2}$, between a double precision approximation of $\eta_{N}^{256}(x ; 0.04)$, and a highly resolved calculation, $\eta_{30}^{256}(x ; 0.04)$ in quadruple precision. We note that at $\varepsilon=0.04$ the quadruple precision calculation is fully converged at $N=30$ indicating that $\varepsilon=0.04$ is within the disk of convergence of the Taylor series (12).

We point out that through several orders the double precision calculation agrees with the "exact" solution. However, a careful investigation of the "raw data" shows that the two calculations begin to diverge at $N=19$ (where three digits are lost from the $N=18$ calculation) and, furthermore, the rate of divergence is exponential (as becomes visually evident in Fig. 1 at $N=22$ ). As a result of this, the double precision approximation is only able to realize a relative accuracy of about $1 \%$ while the quadruple precision results attain a full order of magnitude better accuracy with just a few more iterations.

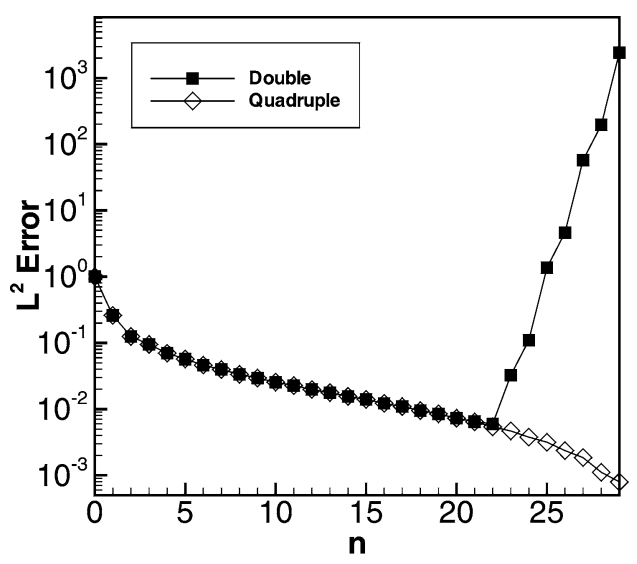

Fig. 1. Comparison of double and quadruple precision computations of $\eta_{N}^{256}$, cf. (13), with a highly resolved solution (quadruple precision calculation with $\left.N_{1}=256, N=30\right)$. Relative error is measured in the $L^{2}$ norm $\left(N_{1}=256,0 \leqslant N \leqslant 29, \varepsilon=0.04, \sigma=0, h=\infty, g=1, \kappa=3\right.$, $\left.c_{0}=1 / \sqrt{3}\right)$. 


\subsection{Transformed field expansions}

The computations of Section 3.1 reveal the subtle cancellations present in the FE recursions for all $N$ and which increase in severity as $N$ is increased. As we noted in [1], the presence of these cancellations precludes the most natural and direct approach to estimating the convergence of the series (12), e.g. through bounds of the form

$$
\left\|\varphi_{n}\right\|_{X} \leqslant C B^{n} .
$$

However, as we demonstrated there, a direct estimation of the terms in (12) can be effected if a change of variables is completed prior to the perturbation expansion. This change of variables succeeds as it implicitly accounts for all significant cancellations (see [1]); this suggests that a numerical implementation of these new recursions should display significant gains in stability over the classical FE formulas (see Fig. 2). As we show in the next section, this conjecture can be translated into a practical and accurate numerical scheme that significantly improves on classical perturbation methods. For the sake of clarity and completeness in substantiating these claims we shall next review the details behind this new approach which we shall refer to as the method of "Transformed Field Expansions" (TFE).

To this end we begin by introducing the change of variables

$$
x^{\prime}=x, \quad y^{\prime}=a\left(\frac{y-\eta}{a+\eta}\right),
$$

which maps the domain $S_{a, \eta}$ to the strip $S_{a, 0}$. (This transform is well-known in many branches of physics, e.g. as $\sigma$-coordinates in oceanography [34].) Defining

$$
u\left(x^{\prime}, y^{\prime}\right)=\varphi\left(x^{\prime},(a+\eta) y^{\prime} / a+\eta\right)
$$

Eqs. (6) become, upon dropping primes,

$$
\begin{array}{ll}
\Delta u=F(x, y), & -a<y<0, \\
\partial_{y} u(x,-a)-T u(x,-a)=J(x), & \\
{\left[c_{0} \cdot \nabla_{x}\right] \eta-\partial_{y} u=Q(x)} & \text { at } y=0, \\
{\left[c_{0} \cdot \nabla_{x}\right] u+\left[g-\sigma \Delta_{x}\right] \eta=R(x)} & \text { at } y=0,
\end{array}
$$

where

$$
\begin{aligned}
F(x, y)=\operatorname{div}_{x}\left[F^{(1)}(x, y)\right]+\partial_{y} F^{(2)}(x, y)+F^{(3)}(x, y) \\
F^{(1)}=-\frac{2}{a} \eta \nabla_{x} u-\frac{1}{a^{2}} \eta^{2} \nabla_{x} u+\frac{a+y}{a} \nabla_{x} \eta \partial_{y} u+\frac{a+y}{a^{2}} \eta \nabla_{x} \eta \partial_{y} u \\
F^{(2)}=\frac{a+y}{a} \nabla_{x} \eta \cdot \nabla_{x} u+\frac{a+y}{a^{2}} \eta \nabla_{x} \eta \cdot \nabla_{x} u-\frac{(a+y)^{2}}{a^{2}}\left|\nabla_{x} \eta\right|^{2} \partial_{y} u \\
F^{(3)}=\frac{1}{a} \nabla_{x} \eta \cdot \nabla_{x} u+\frac{1}{a^{2}} \eta \nabla_{x} \eta \cdot \nabla_{x} u-\frac{a+y}{a^{2}}\left|\nabla_{x} \eta\right|^{2} \partial_{y} u \\
\begin{aligned}
J= & \frac{1}{a} \eta T u \\
Q= & -\left(c-c_{0}\right) \cdot \nabla_{x} \eta-\frac{1}{a}\left(c-c_{0}\right) \cdot \eta \nabla_{x} \eta-\nabla_{x} \eta \cdot \nabla_{x} u-\frac{1}{a} \eta \nabla_{x} \eta \cdot \nabla_{x} u+\left|\nabla_{x} \eta\right|^{2} \partial_{y} u \\
R= & -\left(c-c_{0}\right) \cdot \nabla_{x} u-\frac{2}{a} c \cdot \eta \nabla_{x} u-\frac{1}{a^{2}} c \cdot \eta^{2} \nabla_{x} u+c \cdot \nabla_{x} \eta \partial_{y} u+\frac{1}{a} c \cdot \eta \nabla_{x} \eta \partial_{y} u-\frac{2}{a} g \eta^{2}-\frac{1}{a} g \eta^{3} \\
& +\frac{2}{a} \sigma \eta \Delta_{x} \eta+\frac{1}{a^{2}} \sigma \eta^{2} \Delta \eta-\frac{1}{2}\left|\nabla_{x} u\right|^{2}-\frac{1}{a} \eta\left|\nabla_{x} u\right|^{2}-\frac{1}{2 a^{2}} \eta^{2}\left|\nabla_{x} u\right|^{2}+\nabla_{x} \eta \cdot \nabla_{x} u \partial_{y} u+\frac{1}{a} \eta \nabla_{x} \eta \cdot \nabla_{x} u \partial_{y} u \\
& -\frac{1}{2}\left|\nabla_{x} \eta\right|^{2}\left(\partial_{y} u\right)^{2}-\frac{1}{2}\left(\partial_{y} u\right)^{2}+\sigma H(\eta)+\frac{2}{a} \sigma \eta H(\eta)+\frac{1}{a^{2}} \sigma \eta^{2} H(\eta)
\end{aligned}
\end{aligned}
$$

To solve (16) we expand:

$$
u(x, y, \varepsilon)=\sum_{n=1}^{\infty} u_{n}(x, y) \varepsilon^{n}, \quad \eta(x, \varepsilon)=\sum_{n=1}^{\infty} \eta_{n}(x) \varepsilon^{n}, \quad c(\varepsilon)=\sum_{n=0}^{\infty} c_{n} \varepsilon^{n},
$$


and insert into (16); this results in the following set of problems:

$$
\begin{array}{ll}
\Delta u_{n}=F_{n}(x, y), & -a<y<0, \\
\partial_{y} u_{n}(x,-a)-T u_{n}(x,-a)=J_{n}(x), & \\
{\left[c_{0} \cdot \nabla_{x}\right] \eta_{n}-\partial_{y} u_{n}=Q_{n}(x)-\left[c_{n-1} \cdot \nabla_{x}\right] \eta_{1}} & \text { at } y=0, \\
{\left[c_{0} \cdot \nabla_{x}\right] u_{n}+\left[g-\sigma \Delta_{x}\right] \eta_{n}=R_{n}(x)-\left[c_{n-1} \cdot \nabla_{x}\right] u_{1}} & \text { at } y=0 .
\end{array}
$$

The terms on the right-hand side of (18) are

$$
\begin{aligned}
& F_{n}(x, y)=\operatorname{div}_{x}\left[F_{n}^{(1)}(x, y)\right]+\partial_{y} F_{n}^{(2)}(x, y)+F_{n}^{(3)}(x, y), \\
& F_{n}^{(1)}=-\frac{2}{a} \sum_{l=1}^{n-1} \eta_{l} \nabla_{x} u_{n-l}-\frac{1}{a^{2}} \sum_{m=2}^{n-1} \sum_{l=1}^{m-1} \eta_{l} \eta_{m-l} \nabla_{x} u_{n-m} \\
& +\frac{a+y}{a} \sum_{l=1}^{n-1} \nabla_{x} \eta_{l} \partial_{y} u_{n-l}+\frac{a+y}{a^{2}} \sum_{m=2}^{n-1} \sum_{l=1}^{m-1} \eta_{l} \nabla_{x} \eta_{m-l} \partial_{y} u_{n-m}, \\
& F_{n}^{(2)}=\frac{a+y}{a} \sum_{l=1}^{n-1} \nabla_{x} \eta_{l} \cdot \nabla_{x} u_{n-l}+\frac{a+y}{a^{2}} \sum_{m=2}^{n-1} \sum_{l=1}^{m-1} \eta_{l} \nabla_{x} \eta_{m-l} \cdot \nabla_{x} u_{n-m} \\
& -\frac{(a+y)^{2}}{a^{2}} \sum_{m=2}^{n-1} \sum_{l=1}^{m-1} \nabla_{x} \eta_{l} \cdot \nabla_{x} \eta_{m-l} \partial_{y} u_{n-m} \\
& F_{n}^{(3)}=\frac{1}{a} \sum_{l=1}^{n-1} \nabla_{x} \eta_{l} \cdot \nabla_{x} u_{n-l}+\frac{1}{a^{2}} \sum_{m=2}^{n-1} \sum_{l=1}^{m-1} \eta_{l} \nabla_{x} \eta_{m-l} \cdot \nabla_{x} u_{n-m} \\
& -\frac{a+y}{a^{2}} \sum_{m=2}^{n-1} \sum_{l=1}^{m-1} \nabla_{x} \eta_{l} \cdot \nabla_{x} \eta_{m-l} \partial_{y} u_{n-m} \\
& J_{n}=\frac{1}{a} \sum_{l=1}^{n-1} \eta_{l} T u_{n-l} \\
& Q_{n}=-\sum_{l=1}^{n-2} c_{l} \cdot \nabla_{x} \eta_{n-l}-\frac{1}{a} \sum_{m=1}^{n-1} \sum_{l=0}^{m-1} c_{l} \cdot \eta_{m-l} \nabla_{x} \eta_{n-m}-\sum_{l=1}^{n-1} \nabla_{x} \eta_{l} \cdot \nabla_{x} u_{n-l} \\
& -\frac{1}{a} \sum_{m=2}^{n-1} \sum_{l=1}^{m-1} \eta_{l} \nabla_{x} \eta_{m-l} \cdot \nabla_{x} u_{n-m}+\sum_{m=2}^{n-1} \sum_{l=1}^{m-1} \nabla_{x} \eta_{l} \cdot \nabla_{x} \eta_{m-l} \partial_{y} u_{n-m} \\
& R_{n}=-\sum_{l=1}^{n-2} c_{l} \cdot \nabla_{x} u_{n-l}-\frac{2}{a} \sum_{m=1}^{n-1} \sum_{l=0}^{m-1} c_{l} \cdot \eta_{m-l} \nabla_{x} u_{n-m}-\frac{1}{a^{2}} \sum_{t=2}^{n-1} \sum_{m=1}^{t-1} \sum_{l=0}^{m-1} c_{l} \cdot \eta_{m-l} \eta_{t-m} \nabla_{x} u_{n-t} \\
& +\sum_{m=1}^{n-1} \sum_{l=0}^{m-1} c_{l} \cdot \nabla_{x} \eta_{m-l} \partial_{y} u_{n-m}+\frac{1}{a} \sum_{t=2}^{n-1} \sum_{m=1}^{t-1} \sum_{l=0}^{m-1} c_{l} \cdot \eta_{m-l} \nabla_{x} \eta_{t-m} \partial_{y} u_{n-t}-\frac{2}{a} g \sum_{l=1}^{n-1} \eta_{l} \eta_{n-l} \\
& -\frac{1}{a} g \sum_{m=2}^{n-1} \sum_{l=1}^{m-1} \eta_{l} \eta_{m-l} \eta_{n-m}+\frac{2}{a} \sigma \sum_{l=1}^{n-1} \eta_{l} \Delta_{x} \eta_{n-l}+\frac{1}{a^{2}} \sigma \sum_{m=2}^{n-1} \sum_{l=1}^{m-1} \eta_{l} \eta_{m-l} \Delta \eta_{n-m}-\frac{1}{2} \sum_{l=1}^{n-1} \nabla_{x} u_{l} \cdot \nabla_{x} u_{n-l} \\
& -\frac{1}{a} \sum_{m=2}^{n-1} \sum_{l=1}^{m-1} \eta_{l} \nabla_{x} u_{m-l} \cdot \nabla_{x} u_{n-m}-\frac{1}{2 a^{2}} \sum_{t=3}^{n-1} \sum_{m=2}^{t-1} \sum_{l=1}^{m-1} \eta_{l} \eta_{m-l} \nabla_{x} u_{t-m} \cdot \nabla_{x} u_{n-t} \\
& +\sum_{m=2}^{n-1} \sum_{l=1}^{m-1} \nabla_{x} \eta_{l} \cdot \nabla_{x} u_{m-l} \partial_{y} u_{n-m}+\frac{1}{a} \sum_{t=3}^{n-1} \sum_{m=2}^{t-1} \sum_{l=1}^{m-1} \eta_{l} \nabla_{x} \eta_{m-l} \cdot \nabla_{x} u_{t-m} \partial_{y} u_{n-t}
\end{aligned}
$$




$$
\begin{aligned}
& -\frac{1}{2} \sum_{t=3}^{n-1} \sum_{m=2}^{t-1} \sum_{l=1}^{m-1} \nabla_{x} \eta_{l} \cdot \nabla_{x} \eta_{m-l} \partial_{y} u_{t-m} \partial_{y} u_{n-t}-\frac{1}{2} \sum_{l=1}^{n-1} \partial_{y} u_{l} \partial_{y} u_{n-l} \\
& +\sigma H_{n}(\eta)+\frac{2}{a} \sigma \sum_{l=2}^{n-1} H_{l} \eta_{n-l}+\frac{1}{a^{2}} \sigma \sum_{m=3}^{n-1} \sum_{l=2}^{m-1} H_{l} \eta_{m-l} \eta_{n-m} .
\end{aligned}
$$

The TFE procedure involves solving (18) recursively up to order $N$ starting at order one with solutions of the form (11):

$$
\begin{aligned}
& \eta_{1}(x)=\sum_{j=1}^{d-1} \rho_{j}\left(c_{0} \cdot \kappa_{j}\right) \cos \left(\kappa_{j} \cdot x\right), \\
& \varphi_{1}(x, y)=\sum_{j=1}^{d-1} \rho_{j}\left(g+\sigma\left|\kappa_{j}\right|^{2}\right) \frac{\cosh \left(\left|\kappa_{j}\right|(y+h)\right)}{\cosh \left(h\left|\kappa_{j}\right|\right)} \sin \left(\kappa_{j} \cdot x\right),
\end{aligned}
$$

where we may set $\rho_{1}=1$, cf. (11). For $n>1$, periodic lateral boundary conditions imply that solutions can be expressed as

$$
u_{n}(x, y)=\sum_{k \in \Gamma^{\prime}} \hat{u}_{n}(k, y) \mathrm{e}^{\mathrm{i} k \cdot x}, \quad \eta_{n}(x)=\sum_{k \in \Gamma^{\prime}} \hat{\eta}_{n}(k) \mathrm{e}^{\mathrm{i} k \cdot x},
$$

and (18) transforms to an elliptic two-point boundary value problem at each wavenumber $k \in \Gamma^{\prime}$,

$$
\begin{aligned}
& {\left[\partial_{y}^{2}-|k|^{2}\right] \hat{u}_{n}(k, y)=\widehat{F}_{n}(k, y), \quad-a<y<0,} \\
& \partial_{y} \hat{u}_{n}(k,-a)-|k| \tanh ((h-a)|k|) \hat{u}_{n}(k,-a)=\hat{J}_{n}(k), \\
& {\left[\mathrm{i} c_{0} \cdot k\right] \hat{\eta}_{n}(k)-\partial_{y} \hat{u}_{n}(k, 0)=\widehat{Q}_{n}(k)-\left[\mathrm{i} c_{n-1} \cdot k\right] \hat{\eta}_{1}(k),} \\
& {\left[\mathrm{i} c_{0} \cdot k\right] \hat{u}_{n}(k, 0)+\left[g+\sigma|k|^{2}\right] \hat{\eta}_{n}(k)=\widehat{R}_{n}(k)-\left[\mathrm{i} c_{n-1} \cdot k\right] \hat{u}_{1}(k, 0) .}
\end{aligned}
$$

For each $k \neq 0, \kappa_{j}$ this problem is uniquely solvable. The ambiguity at $k=0$ stems from the non-uniqueness of the velocity potential and can therefore be readily resolved, e.g. by requiring that

$$
\int_{P(\Gamma)} \partial_{y} u(x,-h)=0 .
$$

The singularity in the linear system at wavenumbers $k=\kappa_{j}$, on the other hand, arises from the choice of $c_{0}$. The unspecified velocity $c_{n-1}$ can be used to determine a solution via a system of equations. To derive these equations consider the prototype elliptic system, cf. (18),

$$
\begin{array}{ll}
\Delta u=F(x, y), & -a<y<0, \\
\partial_{y} u(x,-a)-T u(x,-a)=J(x), & \\
{\left[c_{0} \cdot \nabla_{x}\right] \eta-\partial_{y} u=Q(x)-\left[\mu \cdot \nabla_{x}\right] f} & \text { at } y=0, \\
{\left[c_{0} \cdot \nabla_{x}\right] u+\left[g-\sigma \Delta_{x}\right] \eta=R(x)-\left[\mu \cdot \nabla_{x}\right] \xi} & \text { at } y=0,
\end{array}
$$

where $f, \xi$, and $\mu$ are intended to represent $\eta_{1}, u_{1}(x, 0)$, and $c_{n-1}$ respectively. The solution of (21a), (21b), and periodic boundary conditions is

$$
u(x, y)=\sum_{k \in \Gamma^{\prime}} \hat{u}(k, y) \mathrm{e}^{\mathrm{i} k \cdot x},
$$

where (for $k \neq 0$ )

$$
\hat{u}(k, y)=D_{k} \frac{\cosh (|k|(y+h))}{\cosh (h|k|)}+\frac{1}{|k|} \sinh (|k|(y+h)) \cosh (|k|(h-a)) \hat{J}(k)+\frac{1}{|k|} I_{s}(y),
$$

and

$$
I_{s}(y)=\int_{-a}^{y} \sinh (|k|(y-s)) \widehat{F}(k, s) \mathrm{d} s, \quad I_{c}(y)=\int_{-a}^{y} \cosh (|k|(y-s)) \widehat{F}(k, s) \mathrm{d} s .
$$


Eqs. (21c) and (21d) demand that

$$
\left(\begin{array}{cc}
-|k| \tanh (h|k|) & \mathrm{i} c_{0} \cdot k \\
\mathrm{i} c_{0} \cdot k & g+\sigma|k|^{2}
\end{array}\right)\left(\begin{array}{c}
D_{k} \\
\hat{\eta}(k)
\end{array}\right)=\left(\begin{array}{c}
\Phi_{k}-(\mathrm{i} \mu \cdot k) \hat{f}(k) \\
\Psi_{k}-(\mathrm{i} \mu \cdot k) \hat{\xi}(k)
\end{array}\right),
$$

where

$$
\begin{aligned}
& \Phi_{k}=\widehat{Q}(k)+\cosh (h|k|) \cosh ((h-a)|k|) \hat{J}(k)+I_{c}(0), \\
& \Psi_{k}=\widehat{R}(k)-\frac{\mathrm{i} c_{0} \cdot k}{|k|} \sinh (h|k|) \cosh ((h-a)|k|) \hat{J}(k)-\frac{\mathrm{i} c_{0} \cdot k}{|k|} I_{S}(0) .
\end{aligned}
$$

Thus, at $k=\kappa_{j}$ we must have that

$$
\kappa_{j} \cdot \mu=\frac{-\left(\mathrm{i} c_{0} \cdot \kappa_{j}\right) \Phi_{\kappa_{j}}-\left|\kappa_{j}\right| \tanh \left(h\left|\kappa_{j}\right|\right) \Psi_{\kappa_{j}}}{\left(c_{0} \cdot \kappa_{j}\right) \hat{\eta}\left(\kappa_{j}\right)-\left|\kappa_{j}\right| \tanh \left(h\left|\kappa_{j}\right|\right) \hat{\xi}\left(\kappa_{j}\right)},
$$

which is well-defined by the choices of $\hat{\eta}\left(\kappa_{j}\right)$ and $\hat{\xi}\left(\kappa_{j}\right)$, cf. (19). This results in the system $K c_{n-1}=r$, where $r_{j}$ is given by the right-hand side of (23), and $K$ is the matrix from Section 2.2.

Of course, with the singularity of (18) at wavenumbers $k=\kappa_{1}, \ldots, \kappa_{d-1}$ comes not only the issue of solvability but also uniqueness. To address the latter we utilize one of two parameterizations, one determined by orthogonality constraints, and the other by conditions on the wave amplitude. The first parameterization specifies that $\hat{\eta}_{n}\left(\kappa_{j}\right)=0$ $(n>1)$ so that $\eta_{n}$ is orthogonal to $\eta_{1}$; then $\hat{u}_{n}\left(\kappa_{j}\right)$ can be computed from (20). However, Schwartz [9] points out that, for two-dimensional traveling waves, this parameterization of the branch of solutions contains a square-root singularity well before the physical singularity at the Stokes critical wave. He proposed an alternative parameterization in terms of (half) the peak-to-trough height,

$$
h \equiv \frac{1}{2}\left[\eta\left(x_{\max }\right)-\eta\left(x_{\min }\right)\right],
$$

where $x_{\max }$ and $x_{\min }$ are the $x$-coordinates of the maximum and minimum values of $\eta_{1}$, respectively. We note that Roberts et al. [20-22] also utilized this parameterization for three-dimensional traveling waves.

To implement this parameterization we note that

$$
\eta(x, h)=\sum_{n=1}^{\infty} \eta_{n}(x) h^{n}
$$

and insert this into the definition (24):

$$
h=\frac{1}{2}\left[\eta_{1}\left(x_{\max }\right)-\eta_{1}\left(x_{\min }\right)\right] h+\sum_{n=2}^{\infty} \frac{1}{2}\left[\eta_{n}\left(x_{\max }\right)-\eta_{n}\left(x_{\min }\right)\right] h^{n} .
$$

So, at order one

$$
1=\frac{1}{2} \sum_{j=1}^{d-1} \rho_{j}\left(\cos \left(\kappa_{j} \cdot x_{\max }\right)-\cos \left(\kappa_{j} \cdot x_{\min }\right)\right),
$$

where the ratios $\rho_{j} / \rho_{1}$ are fixed and any of the $\rho_{j}$ may be adjusted to satisfy this constraint. In addition, we need to enforce,

$$
0=\eta_{n}\left(x_{\max }\right)-\eta_{n}\left(x_{\min }\right), \quad n \geqslant 2,
$$

which ensures that the third term in (25) vanishes. This choice still leaves us freedom to impose $(d-2)$ additional equations on $\hat{\eta}_{n}\left(\kappa_{j}\right)$ which, for example, may take the form

$$
\frac{\hat{\eta}_{n}\left(\kappa_{j}\right)}{\hat{\eta}_{n}\left(\kappa_{1}\right)}=\frac{\hat{\eta}_{1}\left(\kappa_{j}\right)}{\hat{\eta}_{1}\left(\kappa_{1}\right)}
$$

for $j=2, \ldots, d-1$. 


\section{Numerical results}

As we shall show in the following subsections, the TFE method constitutes a reliable, high-order algorithm for the numerical simulation of traveling water wave forms. As we have explained, due to its favorable execution time and lack of significant cancellations, it can produce simulations at a fraction of the cost of classical boundary perturbation methods such as the FE recursions. As a result, physically relevant quantities such as the wave frequency, energy, and shape can be resolved with a great degree of accuracy. To illustrate these characteristics we shall present examples of fully three-dimensional $(d=3)$ calculations.

\subsection{Numerical method}

Eqs. (18) prescribe, almost completely, the TFE algorithm for simulating traveling water waves. To complete the specification of the method in its three-dimensional version we posit an approximate solution of the form:

$$
\begin{aligned}
& u_{N}^{N_{1}, N_{2}, N_{y}}(x, y)=\sum_{n=0}^{N} \sum_{m_{1}=-N_{1} / 2}^{N_{1} / 2-1} \sum_{m_{2}=-N_{2} / 2}^{N_{2} / 2-1} \sum_{l=0}^{N_{y}} \hat{u}_{n}^{m_{1}, m_{2}, l} T_{l}\left(\frac{2 y+a}{a}\right) \mathrm{e}^{\mathrm{i}\left(k_{m}^{1} x_{1}+k_{m}^{2} x_{2}\right)} h^{n}, \\
& \eta_{N}^{N_{1}, N_{2}}(x)=\sum_{n=0}^{N} \sum_{m_{1}=-N_{1} / 2}^{N_{1} / 2-1} \sum_{m_{2}=-N_{2} / 2}^{N_{2} / 2-1} \hat{\eta}_{n}^{m_{1}, m_{2}} \mathrm{e}^{\mathrm{i}\left(k_{m}^{1} x_{1}+k_{m}^{2} x_{2}\right)} h^{n},
\end{aligned}
$$

where $T_{l}$ is the $l$-th Chebyshev polynomial, and $k_{m}=\left(k_{m}^{1}, k_{m}^{2}\right)$ is a wavenumber (in $\left.\Gamma^{\prime}\right)$ indexed by $m=\left(m_{1}, m_{2}\right)$. These are simply inserted into (18) and the Fourier collocation-Chebyshev tau method (see Canuto, Hussaini, Quarteroni, and Zang [35]) provides a linear system of equations, at every order $n$ and wavenumber $k$, which the $\hat{u}_{n}^{m, l}$ and $\hat{\eta}_{n}^{m}$ must satisfy. At wavenumbers $\kappa_{1}$ and $\kappa_{2}$, (23) can be implemented directly, and either of the uniqueness specification schemes (orthogonality or wave-height; see Section 3.2) can be utilized.

As we anticipated, this TFE algorithm displays significantly enhanced stability properties over those of FE. To illustrate this we present, in Fig. 2, results of the TFE algorithm when applied to the two-dimensional example of Fig. 1 showing no loss of accuracy up to order 29.

From (26) it is clear that storage requirements will be $\mathcal{O}\left(N N_{1} N_{2} N_{y}\right)$. Regarding execution time, the "elliptic solve", required in (18a), can be done at every order $n$ and wavenumber $k_{m}$, and it is a two-point boundary value problem with cost $\mathcal{O}\left(N_{y} \log \left(N_{y}\right)\right)$ (see Canuto, Hussaini, Quarteroni, and Zang [35] and Gottlieb and Orszag [36]);

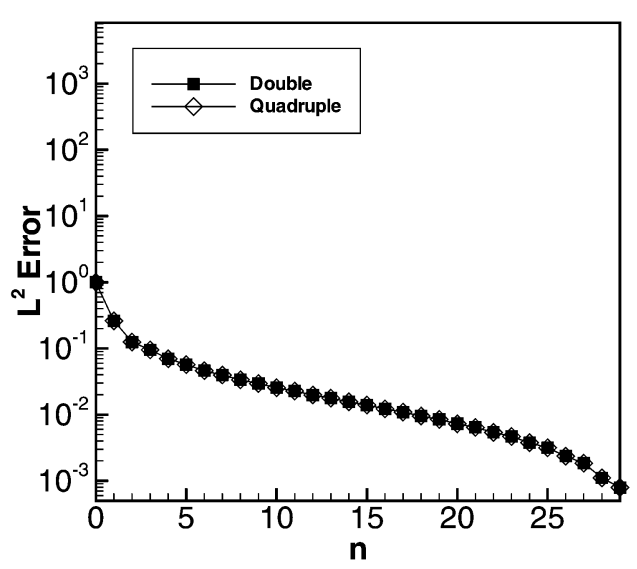

Fig. 2. Comparison of Transformed Field Expansion (double precision) and Field Expansion (quadruple precision) computations of $\eta_{N}^{256}$, cf. (13), with a highly resolved solution (quadruple precision calculation with $\left.N_{1}=256, N=30\right)$. Relative error is measured in the $L^{2}$ norm $\left(N_{1}=256\right.$, $\left.0 \leqslant N \leqslant 29, \varepsilon=0.04, \sigma=0, h=\infty, g=1, \kappa=3, c_{0}=1 / \sqrt{3}\right)$. 
thus the cost of this aspect of the algorithm is $\mathcal{O}\left(N N_{1} N_{2} N_{y} \log \left(N_{y}\right)\right)$. A more subtle bottleneck in execution time comes from the assemblage of the $F_{n}, J_{n}, Q_{n}$, and $R_{n}$ from (18). It would appear that forming $F_{n}$, e.g.

$$
F_{n}^{(1)}=-\frac{1}{a^{2}} \sum_{m=2}^{n-1} \sum_{l=1}^{m-1} \eta_{l} \eta_{m-l} \nabla_{x} u_{n-m}+\cdots
$$

will require, at every order, time proportional to $\mathcal{O}\left(n^{2} N_{1} N_{2} N_{y}\right)$, while the cost of computing $R_{n}$, e.g.

$$
R_{n}=-\frac{1}{2 a^{2}} \sum_{t=3}^{n-1} \sum_{m=2}^{t-1} \sum_{l=1}^{m-1} \eta_{l} \eta_{m-l} \nabla_{x} u_{t-m} \cdot \nabla_{x} u_{n-t}+\cdots
$$

would be $\mathcal{O}\left(n^{3} N_{1} N_{2}\right)$ at every order (note that $R_{n}$ is computed only at the surface so the computational expense is independent of $N_{y}$ ). However, these execution times can be significantly reduced at the expense of some extra storage (still, however, of order $\mathcal{O}\left(N N_{1} N_{2} N_{y}\right)$ ). For instance, the evaluation of (27) can be accelerated if, in addition to $\eta_{n}$, we also store the quantities:

$$
A_{n}=\sum_{l=1}^{n-1} \eta_{l} \eta_{n-l}, \quad B_{n}=\sum_{m=2}^{n-1} \sum_{l=1}^{m-1} \eta_{l} \eta_{m-l} \nabla_{x} u_{n-m}=\sum_{l=1}^{n-1} A_{l} \nabla_{x} u_{n-l} .
$$

Indeed, with these additions the evaluation can be effected as

$$
R_{n}=-\frac{1}{2 a^{2}} \sum_{l=1}^{n-1} B_{l} \cdot \nabla_{x} u_{n-l}
$$

at a cost $\mathcal{O}\left(n N_{1} N_{2}\right)$ at every order, $0 \leqslant n \leqslant N$. This produces a total execution time for $\left\{F_{N}, J_{N}, Q_{N}, R_{N}\right\}$ of $\mathcal{O}\left(N^{2} N_{1} N_{2} N_{y}\right)$ which can be significantly lower than the $\mathcal{O}\left(N^{3} N_{1}^{2} N_{2}^{2}\right)$ of the FE approach.

\subsection{Convergence of frequency and energy density}

At this point we specialize to the geometry and notation employed by Roberts et al. [20-22] in their simulations of "short-crested waves" (SCW). A short-crested wave is a traveling waveform that is not only periodic in the direction of propagation, but also periodic in the orthogonal horizontal direction. The period in the propagation direction is set to $L / \sin (\theta)$ while the period in the orthogonal direction is $L / \cos (\theta)$. In this way Roberts [20] describes an incident (Stokes) wavetrain of wavelength $L$ fully reflected from a vertical wall; here $\theta$ gives the angle between the direction of propagation of the incident wave and the normal to the wall.

If we choose the $x_{1}$-axis as the direction of propagation and non-dimensionalize by setting $L=2 \pi$, the solutions will be periodic with respect to the lattice

$$
\Gamma_{\theta}=\left\{\gamma=j_{1} a+j_{2} b \mid a=(2 \pi) / \sin (\theta), b=(2 \pi) / \cos (\theta) ; j_{1}, j_{2} \in \mathbf{Z}\right\},
$$

i.e. $\eta(x+\gamma)=\eta(x)$ for all $\gamma \in \Gamma_{\theta}$. These solutions will have (linear) amplitude ratio $\rho=\rho_{1} / \rho_{2}=1$, cf. (11), and there will be a branch of non-trivial solutions for every pair of (linearly independent) wavenumbers $\left(\kappa_{1}, \kappa_{2}\right)$ parameterized by $\varepsilon$ (or $h$ if Schwartz's method is used). We note that our method is not restricted to such patterns and can generate solutions with arbitrary periodicity and amplitude ratios. In fact, as we shall see in Section 5, our method is not restricted to branches of solutions but can, in fact, produce entire surfaces.

Two quantities of physical relevance measured in Roberts and Schwartz [13] are the (temporal) frequency $\omega$, and the energy density $\mathcal{E}$ of the traveling wave. In the present configuration, the velocity is constrained to have zero $x_{2}$ component, i.e. $c=\left(c_{1}, 0\right)$, and thus the frequency is defined by

$$
\omega=c_{1} \sin (\theta) .
$$

The total energy density is the sum of the kinetic and potential energy densities, $\mathcal{E}=\mathcal{K}+\mathcal{P}$, where

$$
\mathcal{K}=\frac{1}{2|P(\Gamma)|} \int_{P(\Gamma)}\left(\left.\varphi\right|_{y=\eta}\right)\left(\left.\partial_{y} \varphi\right|_{y=\eta}-\left.\nabla_{x} \varphi\right|_{y=\eta} \cdot \nabla_{x} \eta\right) \mathrm{d} x
$$


Table 1

Convergence of $\omega_{N}$ and $\mathcal{E}_{N}$, and $\left|\mathcal{R}_{N}\right| \max$ as $N$ is refined using the TFE algorithm $\left(h=0.2, \theta=45^{\circ}, N_{1}=N_{2}=64, N_{y}=48, N=31\right)$

\begin{tabular}{rlll}
\hline$N$ & $\omega_{N}$ & $\mathcal{E}_{N}$ & $\left|\mathcal{R}_{N}\right|_{\max }$ \\
\hline 4 & 1.005196646512916 & 0.009883644568005213 & $4.362998832815271 \times 10^{-5}$ \\
6 & 1.005194641310237 & 0.009878291421105056 & $4.734026055216306 \times 10^{-6}$ \\
8 & 1.005194594102063 & 0.009878231538493739 & $1.905675412585198 \times 10^{-7}$ \\
10 & 1.005194589197477 & 0.009878223320090387 & $1.58898666425232 \times 10^{-8}$ \\
12 & 1.005194589442087 & 0.009878223868258484 & $1.423264868374158 \times 10^{-9}$ \\
14 & 1.005194589397794 & 0.009878223777685075 & $1.396967238068147 \times 10^{-10}$ \\
16 & 1.005194589401453 & 0.009878223785242023 & $1.449392559096929 \times 10^{-11}$ \\
18 & 1.00519458940103 & 0.009878223784370262 & $1.476996476512671 \times 10^{-12}$ \\
20 & 1.005194589401071 & 0.009878223784456151 & $1.496242366116896 \times 10^{-13}$ \\
22 & 1.005194589401067 & 0.009878223784447264 & $1.706707691839782 \times 10^{-14}$ \\
24 & 1.005194589401067 & 0.009878223784448069 & $7.186959360971912 \times 10^{-15}$ \\
26 & 1.005194589401067 & 0.009878223784448 & $1.367482516112517 \times 10^{-14}$ \\
28 & 1.005194589401067 & 0.009878223784447996 & $3.007837034996186 \times 10^{-14}$ \\
30 & 1.005194589401067 & 0.009878223784447993 & $6.668450514002444 \times 10^{-14}$ \\
\hline
\end{tabular}

$$
\mathcal{P}=\frac{1}{2|P(\Gamma)|} \int_{P(\Gamma)} \eta^{2} \mathrm{~d} x
$$

Also measured in Roberts and Schwartz [13] is the residual in the Bernoulli condition

$$
\mathcal{R}=\left[c \cdot \nabla_{x}\right] \varphi+\frac{1}{2}|\nabla \varphi|^{2}+\left[g-\sigma \Delta_{x}\right] \eta-\sigma H(\eta) \quad \text { at } y=\eta .
$$

This residual, measured in the maximum norm, was interpreted as a "semi-independent error estimate".

Inspired by the computations in Roberts and Schwartz [13], the convergence of

$$
\omega_{N}=\sum_{n=0}^{N} \omega_{n} h^{n}, \quad \mathcal{E}_{N}=\sum_{n=0}^{N} \mathcal{E}_{n} h^{n}, \quad\left|\mathcal{R}_{N}\right|_{\max }=\max _{x \in P(\Gamma)}\left|\sum_{n=0}^{N} \mathcal{R}_{n}(x) h^{n}\right|,
$$

were studied for $h=0.2$ as $N$ was increased. Table 1 summarizes the results for $\theta=45^{\circ}$ derived from a TFE approximation with $N_{1}=N_{2}=64, N_{y}=48$, and $N=31$.

We note that throughout the range of orders, this algorithm consistently delivers increasing accuracy as $N$ is increased. In particular, the estimate of $\omega_{N}$ has full double precision accuracy by $N=22$, while $\mathcal{E}_{N}$ has fifteen digits of accuracy at $N=30$. The magnitude of the residual consistently decreases through all orders and gives us confidence that our calculations are converging to a solution of the Euler equations.

\subsection{Plots of prototypical solutions}

In this subsection we display surface and contour plots of typical traveling wave solutions. To display some of the canonical features of short-crested, three-dimensional traveling surface patterns we have chosen three values of the parameter $\theta$ (in water of infinite depth) for sample calculations. In all calculations the numerical parameters were set to $N_{1}=N_{2}=64, N_{y}=48$, and $N=31$.

Beginning with $\theta=45^{\circ}$, we have selected two values of the parameter $h, h_{1}=0.21$ and $h_{2}=0.43$, which are meant to represent "moderately nonlinear" and "highly nonlinear" profiles. In Fig. 3 we plot the traveling wave surface $\eta$ for these two values of $h$. For these two surfaces, the actual height (measured in the maximum norm) is $\left|\eta\left(x ; h_{1}\right)\right|_{\max }=0.224997$, and $\left|\eta\left(x ; h_{2}\right)\right|_{\max }=0.487929$, respectively. In Fig. 4 we give a contour plot of these two surfaces where two periods in both the $x_{1}$ and $x_{2}$ direction are plotted to give a flavor for the periodic wave-field they represent. In these plots we point out the "diamond-like" shape of the wave due to the symmetries of this configuration; however, we also point out the significant nonlinearity in these forms with the wide, shallow troughs, and (relatively) compact, steep crests (cf. Fig. 3). 


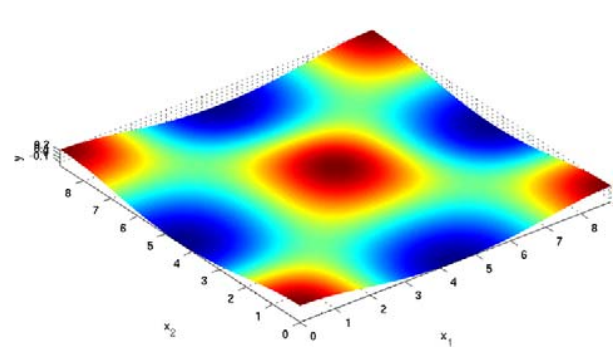

(a)

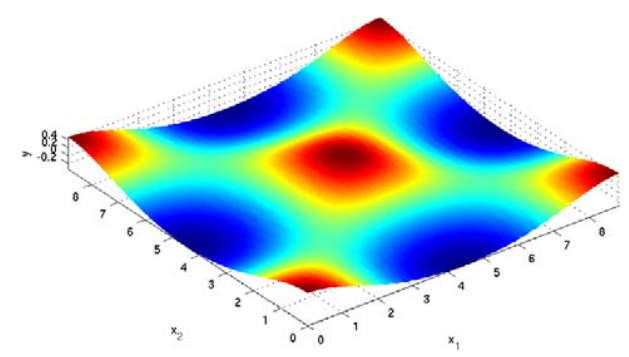

(b)

Fig. 3. Surface plots of two ocean profiles with $\theta=45^{\circ}$ with $h=0.21,0.43$ respectively. (a) Medium, (b) large.

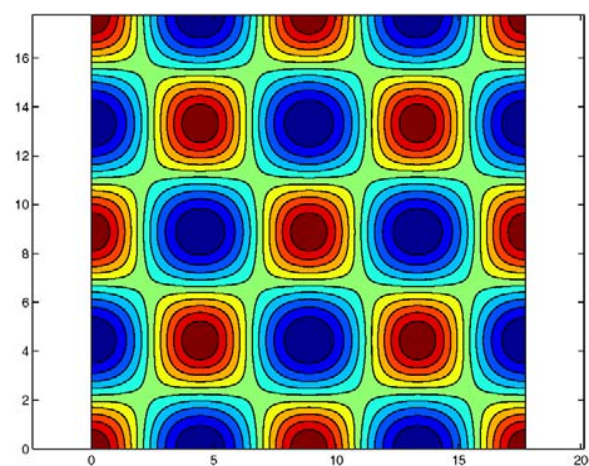

(a)

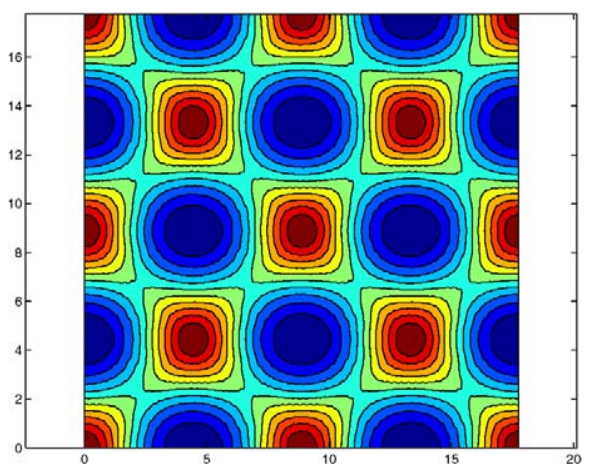

(b)

Fig. 4. Contour plots of two ocean profiles with $\theta=45^{\circ}$ with $h=0.21,0.43$ respectively. (a) Medium, (b) large.

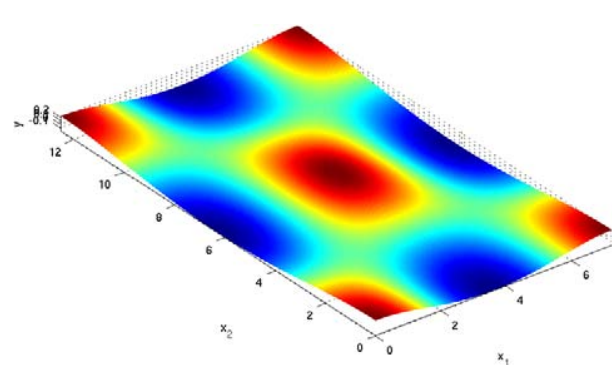

(a)

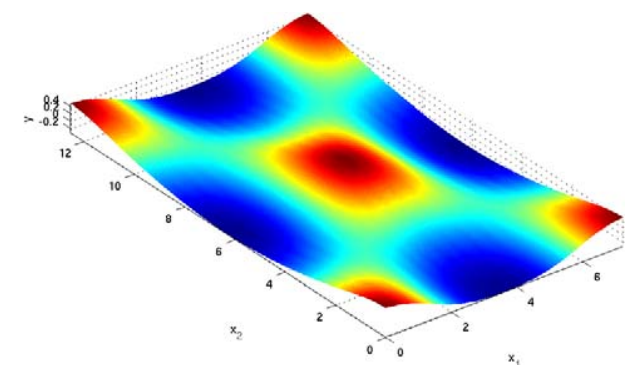

(b)

Fig. 5. Surface plots of two ocean profiles with $\theta=60^{\circ}$ with $h=0.21,0.42$ respectively. (a) Medium, (b) large.

Turning to $\theta=60^{\circ}$, we have again selected two values of the parameter $h, h_{1}=0.21$ (moderately nonlinear) and $h_{2}=0.42$ (highly nonlinear). In Fig. 5 we plot the traveling wave surface $\eta$ for these two values of $h$. For these two surfaces the actual height (measured in the maximum norm) is $\left|\eta\left(x ; h_{1}\right)\right|_{\max }=0.228521$, and $\left|\eta\left(x ; h_{2}\right)\right|_{\max }=$ 0.491571 , respectively. In Fig. 6 we give contour plots of these two surfaces where, again, two periods are pictured. In contrast with the "diamond-like" waveforms of the solutions above, these surfaces display the "rectangular" or "hexagonal" forms characteristic of higher values of $\theta$ (see e.g. Nicholls [16,17], and Craig and Nicholls [18]). Again, in both plots the significant nonlinearity is evident in the long, shallow troughs and sharp, steep crests.

Finally, we consider $\theta=75^{\circ}$. Again we study the traveling wave solutions via our numerical scheme at two values of the perturbation parameter $h, h_{1}=0.2$ and $h_{2}=0.41$. In Fig. 7 we plot the traveling wave surface $\eta$ for $h_{1}$ and $h_{2}$, and the actual heights of these profiles are $|\eta|_{\max }=0.226086$, and $|\eta|_{\max }=0.542079$, respectively. In Fig. 8 we give contour plots of these two surfaces where, again, two periods are pictured. Here the rectangular/hexagonal form of the traveling waves is even more pronounced than in the case $\theta=60^{\circ}$. 


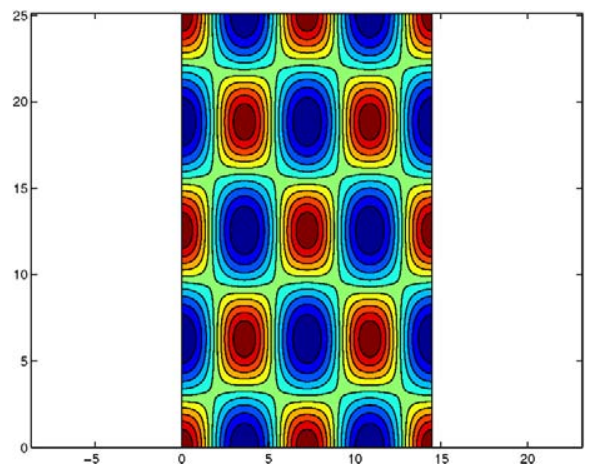

(a)

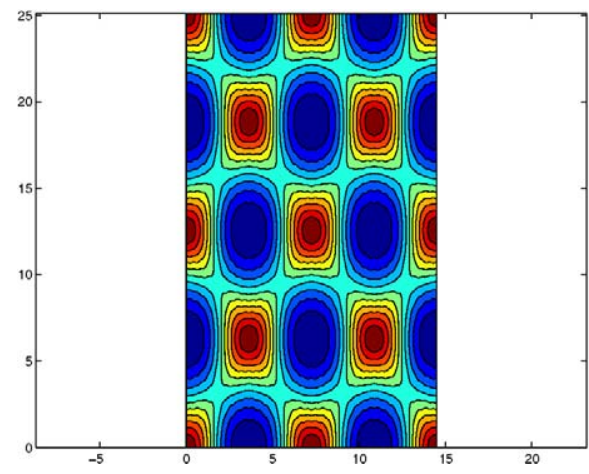

(b)

Fig. 6. Contour plots of two ocean profiles with $\theta=60^{\circ}$ with $h=0.21,0.42$ respectively. (a) Medium, (b) large.

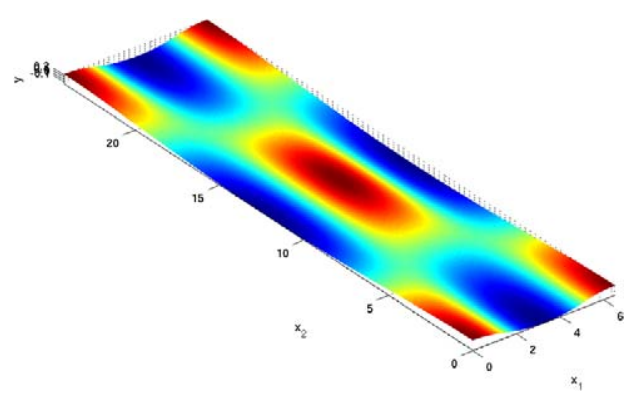

(a)

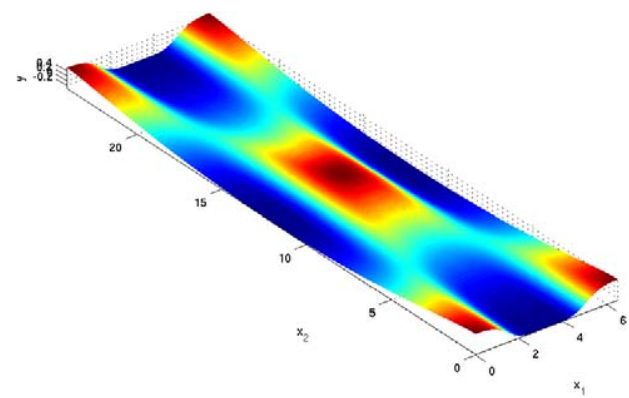

(b)

Fig. 7. Surface plots of two ocean profiles with $\theta=75^{\circ}$ with $h=0.2,0.41$ respectively. (a) Medium, (b) large.

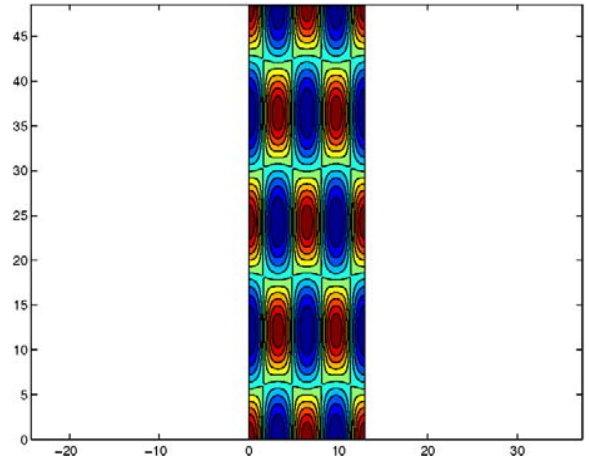

(a)

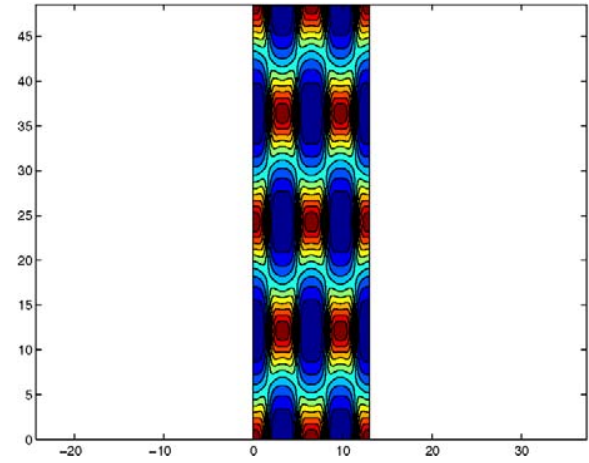

(b)

Fig. 8. Contour plots of two ocean profiles with $\theta=75^{\circ}$ with $h=0.2,0.41$ respectively. (a) Medium, (b) large.

\subsection{Padé enhancement}

All the calculations above were performed with direct summation of the Taylor series (12). A question arises then as to the possibility of accelerating the convergence of these series through alternative summation mechanisms. Most notable among these is the use of Padé approximants [37], whereby the series are approximated by rational functions. As is well-known, this technique is particularly useful when singularities of the analytic extension to the complex plane of the quantities of interest are well separated from the evaluation point (e.g. when such singularities have nonzero imaginary parts and evaluations are effected on the real axis).

In the present case, however, Padé approximation is only marginally useful, especially for highly nonlinear waveforms. Indeed, for the examples above the singularity closest to the origin, as predicted by Padé approximation, 


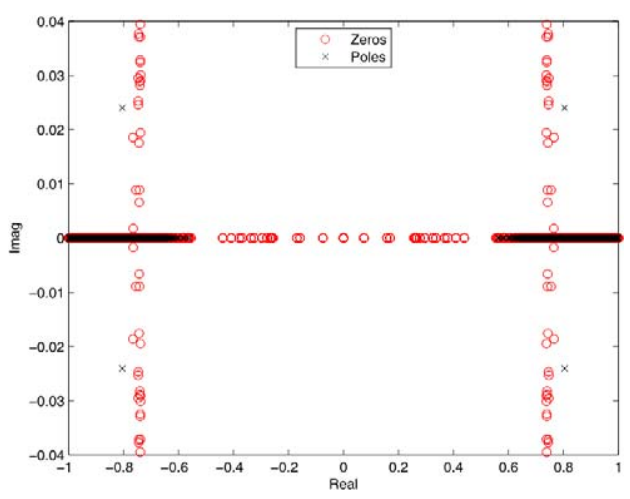

(a)

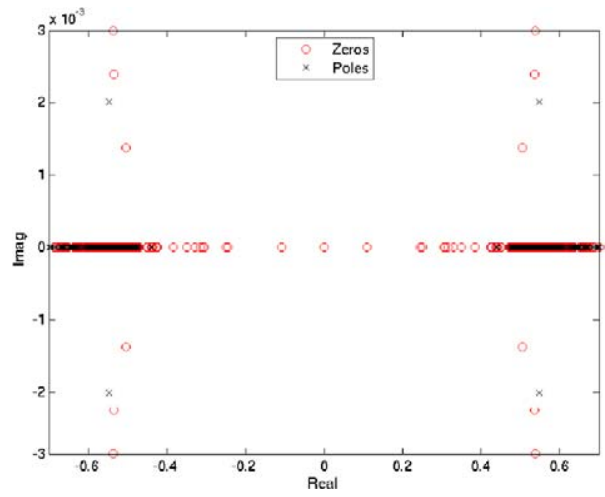

(b)

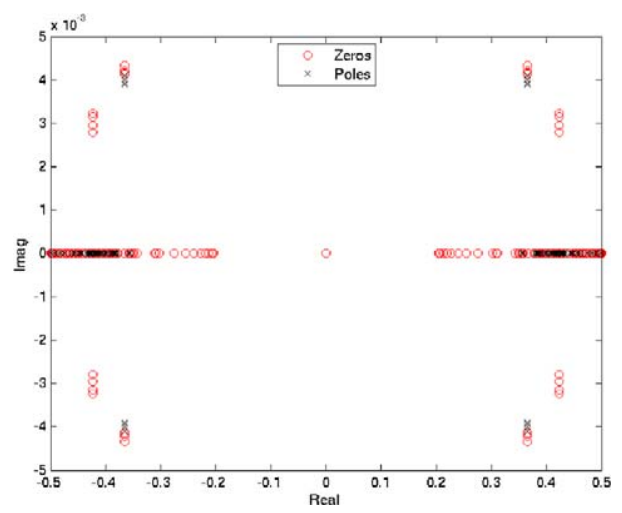

(c)

Fig. 9. Plot of uncanceled zeroes (circles) and poles (crosses) of $\eta\left(x_{j}\right)$ for all gridpoints $x_{j}$, cf. (12), as computed by Padé approximation. A pole (denominator zero) and a zero (numerator zero) are canceled if their distance is less than a tolerance $\tau$ (in this case $\tau=10^{-4}$ ) in the Euclidean norm. (a) $\theta=45^{\circ}$, (b) $\theta=60^{\circ}$, (c) $\theta=75^{\circ}$.

consistently lies on the real axis; see Fig. 9. Moreover, the heights $h_{2}$ in each of the examples of "Large" waveforms is relatively close to this singularity. More quantitatively, the use of Padé approximation in these cases allows for non-singular results up to heights $h_{3}=0.56,0.46,0.41$ (cf. Fig. 9); when applied to the heights $h_{2}$ above, however, Padé approximation produces no distinguishable improvement. As evidence of this, when the maximum amplitude was computed for waveforms at $h_{2}$ and $N$ was refined from zero to 31, Padé summation never consistently delivered more than two extra digits of accuracy.

\section{Surfaces of solutions}

It is clear from the recursions derived in [1] that the perturbation parameter $\varepsilon$ need not be restricted to one dimension, and may, in fact, be viewed as a $(d-1)$-dimensional real quantity. In three dimensions this amounts to $\varepsilon=\left(\varepsilon_{1}, \varepsilon_{2}\right)$, which makes transparent the realization made in Craig and Nicholls [29] (and further elucidated in Craig and Nicholls [18]) that three-dimensional traveling water waves come in surfaces rather than simply in curves. Indeed, as is shown in [1], these surfaces depend analytically on the parameter $\left(\varepsilon_{1}, \varepsilon_{2}\right) \in \mathbb{R}^{2}$; in fact, these solutions are jointly analytic in parameter and spatial variable.

In most aspects the TFE recursions are easily generalized to the case of a parameter $\left(\varepsilon_{1}, \varepsilon_{2}\right) \in \mathbb{R}^{2}$. The change of variables (14) is identical, while the expansions (17) are trivially generalized, e.g.

$$
\varphi\left(x, y, \varepsilon_{1}, \varepsilon_{2}\right)=\sum_{|n| \geqslant 1} \varphi_{n}(x, y) \varepsilon_{1}^{n_{1}} \varepsilon_{2}^{n_{2}}
$$


where $n=\left(n_{1}, n_{2}\right) \in \mathbf{Z}^{2}$ is a multi-index. At every order $|n| \equiv n_{1}+n_{2}=N$, one solves (18), where, e.g.

$$
F_{n}^{(3)}=\frac{1}{a} \sum_{|l|=1}^{|n|-1} \nabla_{x} \eta_{l} \cdot \nabla_{x} u_{n-l}+\frac{1}{a^{2}} \sum_{|m|=2}^{|n|-1} \sum_{|l|=1}^{|m|-1} \eta_{l} \nabla_{x} \eta_{m-l} \cdot \nabla_{x} u_{n-m}-\frac{a+y}{a^{2}} \sum_{|m|=2}^{|n|-1} \sum_{|l|=1}^{|m|-1} \nabla_{x} \eta_{l} \cdot \nabla_{x} \eta_{m-l} \partial_{y} u_{n-m} .
$$

To begin this TFE procedure, solutions of the homogeneous equations at order $|n|=1$ must be specified. As we have throughout, we begin with two wavenumbers $\kappa_{1}, \kappa_{2} \in \Gamma^{\prime}$ and select the unique (up to sign) velocity $c_{0} \in \mathbb{R}^{2}$ such that $\Lambda_{\sigma}\left(c_{0}, \kappa_{j}\right)=0$ (see Section 2.2). We are now free to choose solutions akin to (19), e.g.

$$
\eta_{1,0}(x)=\rho_{1,0}\left(c_{0} \cdot \kappa_{1}\right) \cos \left(\kappa_{1} \cdot x+\theta_{1,0}\right), \quad \eta_{0,1}(x)=\rho_{0,1}\left(c_{0} \cdot \kappa_{2}\right) \cos \left(\kappa_{2} \cdot x+\theta_{0,1}\right),
$$

and, again, without loss of generality we can set $\theta_{l}=0(|l|=1)$. The expansion now becomes

$$
\eta\left(x, \varepsilon_{1}, \varepsilon_{2}\right)=\varepsilon_{1} \rho_{1,0}\left(c_{0} \cdot \kappa_{1}\right) \cos \left(\kappa_{1} \cdot x\right)+\varepsilon_{2} \rho_{0,1}\left(c_{0} \cdot \kappa_{2}\right) \cos \left(\kappa_{2} \cdot x\right)+\mathcal{O}\left(|\varepsilon|^{2}\right),
$$

and it is clear that we can specify an entire surface of solutions by varying $\varepsilon_{1}$ and $\varepsilon_{2}$ independently. In particular, $\varepsilon_{1}=0$ or $\varepsilon_{2}=0$ correspond to two-dimensional Stokes waves, while $\varepsilon_{1}=\varepsilon_{2}$ recovers the short-crested waves of the previous section.

For orders $|n|=N>1$ the specification of this TFE algorithm is precisely the same as that outlined in Section 3.2 with the exception of the resolution of the singularity in the linear operator $A(c, k)$, see (8), at wavenumbers $k=\kappa_{j}$. As discussed in [1] the resolution of this difficulty must be made at every order $N$ simultaneously, for all $n$ such that $|n|=N$. Another aspect of this generalized TFE approach which deserves mention is the implementation of parameterization by "half wave-height", cf. (24). Following the design philosophy of Schwartz [9] we identify the unique points $a_{\min }, a_{\max }, b_{\min }$, and $b_{\max }$ such that:

(1) $\eta_{1,0}$ is maximized at $a_{\max }$ and minimized at $a_{\min }$,

(2) $\eta_{0,1}$ is maximized at $b_{\max }$ and minimized at $b_{\min }$,

(3) $\eta_{1,0}\left(b_{\max }\right)=\eta_{1,0}\left(b_{\min }\right)$,

(4) $\eta_{0,1}\left(a_{\max }\right)=\eta_{0,1}\left(a_{\min }\right)$,

and define

$$
h_{1} \equiv \frac{1}{2}\left[\eta\left(a_{\max }\right)-\eta\left(a_{\min }\right)\right], \quad h_{2} \equiv \frac{1}{2}\left[\eta\left(b_{\max }\right)-\eta\left(b_{\min }\right)\right] .
$$

Then, following Section 3.2, we expand $\eta$ in powers of $h=\left(h_{1}, h_{2}\right)$

$$
\eta\left(x, h_{1}, h_{2}\right)=\sum_{|n| \geqslant 1} \eta_{n}(x) h_{1}^{n_{1}} h_{2}^{n_{2}},
$$

and insert this into the definitions (31):

$$
\begin{aligned}
& h_{1}=\frac{1}{2}\left[\eta_{1,0}\left(a_{\max }\right)-\eta_{1,0}\left(a_{\min }\right)\right] h_{1}+\sum_{|n| \geqslant 2} \frac{1}{2}\left[\eta_{n}\left(a_{\max }\right)-\eta_{n}\left(a_{\min }\right)\right] h_{1}^{n_{1}} h_{2}^{n_{2}}, \\
& h_{2}=\frac{1}{2}\left[\eta_{0,1}\left(b_{\max }\right)-\eta_{0,1}\left(b_{\min }\right)\right] h_{2}+\sum_{|n| \geqslant 2} \frac{1}{2}\left[\eta_{n}\left(b_{\max }\right)-\eta_{n}\left(b_{\min }\right)\right] h_{1}^{n_{1}} h_{2}^{n_{2}} .
\end{aligned}
$$

So

$$
\begin{aligned}
& 1=\frac{1}{2}\left[\rho_{1,0} \cos \left(\kappa_{1} \cdot a_{\max }\right)-\rho_{1,0} \cos \left(\kappa_{1} \cdot a_{\min }\right)\right], \\
& 1=\frac{1}{2}\left[\rho_{0,1} \cos \left(\kappa_{2} \cdot b_{\max }\right)-\rho_{0,1} \cos \left(\kappa_{2} \cdot b_{\min }\right)\right],
\end{aligned}
$$

where $\rho_{1,0}$ and $\rho_{0,1}$ are adjusted to satisfy these constraints. In addition, we need to enforce,

$$
0=\eta_{n}\left(a_{\max }\right)-\eta_{n}\left(a_{\min }\right)=\eta_{n}\left(b_{\max }\right)-\eta_{n}\left(b_{\min }\right), \quad|n| \geqslant 2,
$$

which ensure that the $\mathcal{O}\left(|h|^{2}\right)$ terms in (32) vanish. 


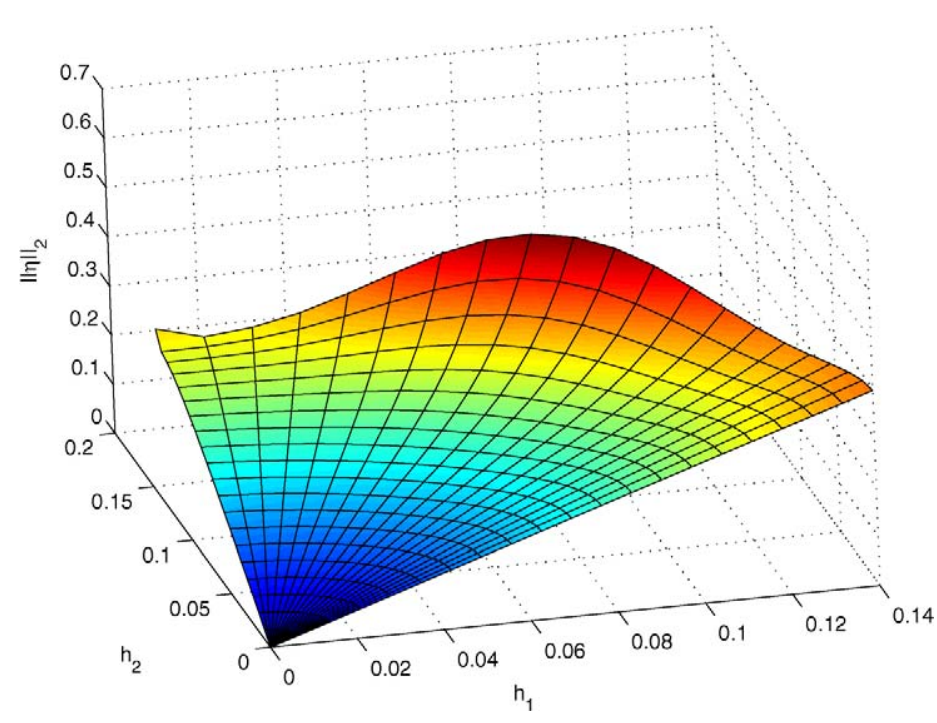

Fig. 10. Plot of $\|\eta\|_{L^{2}}$ versus $\left(h_{1}, h_{2}\right)$ meant to depict the surface of traveling wave solutions. Parameters for this numerical simulation were: $\kappa_{1}=(1,1), \kappa_{2}=(5,-1), N_{1}=16, N_{2}=16, N_{y}=16, N=7, g=1, h=\infty, \theta=60^{\circ}, a=0.5$. The "half wave-height" parameterization (31) was utilized.

We conclude this section with Fig. 10 that depicts the full surface of traveling wave solutions which generically exist for the Euler equations. In this particular calculation the solutions are periodic with respect to the lattice $\Gamma_{\theta},(28)$, with $\theta=60^{\circ}$. Asymmetry in the bifurcation surface is enhanced by the choice of base wavenumbers $\kappa_{1}=(1,1)$ and $\kappa_{2}=(5,-1)$. The physical parameters are $g=1$ and $h=\infty$, and the numerical parameters are $N_{1}=16, N_{2}=16$, $N_{y}=16, N=7$, and $a=0.5$.

\section{Conclusions}

In this paper we have described a new, stable high-order boundary perturbation algorithm for the numerical simulation of traveling water waves. This algorithm is based upon our recent study of analyticity properties of solutions of the Euler equations [1]. Our new scheme compares favorably with classical boundary deformation methods in terms of both conditioning and computational complexity. The improved conditioning results from the avoidance of significant cancellations upon which classical methods rely for convergence. The lower computational cost stems from the nature of the recursions and our implementation of a fast, high-order spectral method to resolve them. Finally, we displayed how the algorithm can be extended to compute the full surfaces (as opposed to simply branches) of traveling wave solutions which we had previously shown to exist.

\section{Acknowledgements}

DPN gratefully acknowledges support from NSF through grants No. DMS-0196452, DMS-0139822, and DMS0406007. FR gratefully acknowledges support from NSF through grant No. DMS-0311763.

\section{References}

[1] D.P. Nicholls, F. Reitich, On analyticity of traveling water waves, Proc. Roy. Soc. London Ser. A 461 (2057) (2005) 1283-1309.

[2] G. Stokes, On the theory of oscillatory waves, Trans. Cambridge Philos. Soc. 8 (1847) 441-473.

[3] W.-T. Tsai, D.K.P. Yue, Computation of nonlinear free-surface flows, Annu. Rev. Fluid Mech. 28 (1996) 249-278.

[4] J.T. Beale, A convergent boundary integral method for three-dimensional water waves, Math. Comp. 70 (235) (2001) 977-1029.

[5] S. Grilli, P. Guyenne, F. Dias, A fully nonlinear model for three-dimensional overturning waves over an arbitrary bottom, Int. J. Numer. Methods Fluids 35 (2001) 829-867.

[6] M. Xue, H. Xü, Y. Liu, D.K.P. Yue, Computations of fully nonlinear three-dimensional wave-wave and wave-body interactions. I. Dynamics of steep three-dimensional waves, J. Fluid Mech. 438 (2001) 11-39. 
[7] Y. Liu, M. Xue, D.K.P. Yue, Computations of fully nonlinear three-dimensional wave-wave and wave-body interactions. II. Nonlinear waves and forces on a body, J. Fluid Mech. 438 (2001) 41-66.

[8] F. Dias, C. Kharif, Nonlinear gravity and capillary-gravity waves, Annu. Rev. Fluid Mech. 31 (1999) 301-346.

[9] L.W. Schwartz, Computer extension and analytic continuation of Stokes' expansion for gravity waves, J. Fluid Mech. 62 (1974) 553-578.

[10] L.W. Schwartz, J.-M. Vanden-Broeck, Numerical solution of the exact equations for capillary-gravity waves, J. Fluid Mech. 95 (1) (1979) 119-139.

[11] M.M. Rienecker, J.D. Fenton, A Fourier approximation method for steady water waves, J. Fluid Mech. 104 (1981) $119-137$.

[12] D. Meiron, P. Saffman, H. Yuen, Calculation of steady three-dimensional deep-water waves, J. Fluid Mech. 124 (1982) $109-121$.

[13] A.J. Roberts, L.W. Schwartz, The calculation of nonlinear short-crested gravity waves, Phys. Fluids 26 (1983) $2388-2392$.

[14] P.G. Saffman, H.C. Yuen, Three-dimensional waves on deep water, in: Advances in Nonlinear Waves, vol. II, Pitman, Boston, MA, 1985, pp. $1-30$.

[15] P.J. Bryant, Doubly periodic progressive permanent waves in deep water, J. Fluid Mech. 161 (1985) $27-42$.

[16] D.P. Nicholls, Traveling water waves: Spectral continuation methods with parallel implementation, J. Comput. Phys. 143 (1) (1998) 224-240.

[17] D.P. Nicholls, On hexagonal gravity water waves, Math. Comput. Simul. 55 (4-6) (2001) 567-575.

[18] W. Craig, D.P. Nicholls, Traveling gravity water waves in two and three dimensions, Eur. J. Mech. B Fluids 21 (6) (2002) $615-641$.

[19] V. Zakharov, Stability of periodic waves of finite amplitude on the surface of a deep fluid, J. Appl. Mech. Techn. Phys. 9 (1968) 190-194.

[20] A.J. Roberts, Highly nonlinear short-crested water waves, J. Fluid Mech. 135 (1983) 301-321.

[21] A.J. Roberts, D.H. Peregrine, Notes on long-crested water waves, J. Fluid Mech. 135 (1983) 323-335.

[22] T.R. Marchant, A.J. Roberts, Properties of short-crested waves in water of finite depth, J. Austral. Math. Soc. Ser. B 29 (1) (1987) $103-125$.

[23] D.P. Nicholls, F. Reitich, Stability of high-order perturbative methods for the computation of Dirichlet-Neumann operators, J. Comput. Phys. 170 (1) (2001) 276-298.

[24] D.P. Nicholls, F. Reitich, A new approach to analyticity of Dirichlet-Neumann operators, Proc. Roy. Soc. Edinburgh Sect. A 131 (6) (2001) 1411-1433.

[25] D.P. Nicholls, F. Reitich, Analytic continuation of Dirichlet-Neumann operators, Numer. Math. 94 (1) (2003) 107-146.

[26] D.P. Nicholls, F. Reitich, Shape deformations in rough surface scattering: Cancellations, conditioning, and convergence, J. Opt. Soc. Am. A 21 (4) (2004) 590-605.

[27] D.P. Nicholls, F. Reitich, Shape deformations in rough surface scattering: Improved algorithms, J. Opt. Soc. Am. A 21 (4) (2004) 606-621.

[28] H. Lamb, Hydrodynamics, sixth ed., Cambridge University Press, Cambridge, 1993.

[29] W. Craig, D.P. Nicholls, Traveling two and three dimensional capillary gravity water waves, SIAM J. Math. Anal. 32 (2) (2000) $323-359$.

[30] K.M. Watson, B.J. West, A transport-equation description of nonlinear ocean surface wave interactions, J. Fluid Mech. 70 (1975) $815-826$.

[31] B.J. West, K.A. Brueckner, R.S. Janda, D.M. Milder, R.L. Milton, A new numerical method for surface hydrodynamics, J. Geophys. Res. 92 (1987) 11803-11824.

[32] D.M. Milder, The effects of truncation on surface-wave Hamiltonians, J. Fluid Mech. 217 (1990) 249-262.

[33] W. Craig, C. Sulem, Numerical simulation of gravity waves, J. Comput. Phys. 108 (1993) 73-83.

[34] N.A. Phillips, A coordinate system having some special advantages for numerical forecasting, J. Atmospheric Sci. 14 (2) (1957) $184-185$.

[35] C. Canuto, M.Y. Hussaini, A. Quarteroni, T.A. Zang, Spectral Methods in Fluid Dynamics, Springer-Verlag, New York, 1988.

[36] D. Gottlieb, S.A. Orszag, Numerical Analysis of Spectral Methods: Theory and Applications, CBMS-NSF Regional Conf. Ser. in Appl. Math., vol. 26, Society for Industrial and Applied Mathematics, Philadelphia, PA, 1977.

[37] G.A. Baker Jr., P. Graves-Morris, Padé Approximants, second ed., Cambridge University Press, Cambridge, 1996. 